\title{
Real-time PCR as a tool for quantitative analysis of PI-PLCB1 gene expression in myelodysplastic syndrome
}

\author{
MATILDE Y. FOLLO ${ }^{1}$, COSTANZA BOSI $^{2}$, CARLO FINELLI $^{2}$, ROBERTA FIUME ${ }^{1}$, IRENE FAENZA ${ }^{1}$, \\ GIULIA RAMAZZOTTI ${ }^{1}$, GIAN CARLO GABOARDI ${ }^{1}$, LUCIA MANZOLI $^{1}$ and LUCIO COCCO $^{1}$ \\ ${ }^{1}$ Department of Anatomical Sciences, Cellular Signalling Laboratory, University of Bologna, via Irnerio 48, 40126 Bologna; \\ ${ }^{2}$ Institute of Hematology and Medical Oncology 'L. e A. Seràgnoli', University of Bologna, Bologna, Italy
}

Received February 3, 2006; Accepted April 29, 2006

\begin{abstract}
Phosphoinositide-specific phospholipase C (PI-PLC) $B 1$ is a key enzyme in nuclear signal transduction, and it is involved in many cellular processes, such as proliferation and differentiation. In particular, the involvement of the PI-PLCB1 gene in erythroid differentiation lead us to investigate this gene in patients affected by high-risk myelodysplastic syndrome (MDS). By using fluorescence in situ hybridization (FISH) analysis, we have previously evidenced that, in MDS patients with normal GTG banding and a fatal outcome, the PI-PLCB1 gene undergoes monoallelic and interstitial deletion. Real-time PCR is characterized by high sensitivity, excellent precision and large dynamic range, and has become the method of choice for quantitative gene expression measurements. In the present study, we have performed a relative quantification real-time polymerase chain reaction (PCR) analysis on all of the MDS patients tested for FISH analysis. Furthermore, we have evaluated the expression of the PI-PLCB1 gene on healthy donors and the HL60 cell line, which is useful for testing the accuracy of the technology because of its low expression of PI-PLCß1. To analyze and quantify the levels of the two different splicing variants of PI-PLCß1 gene (1a and 1b), we have used a TaqMan isoform specific probe. We have seen that all of the MDS patients have higher levels of the PIPLCB1 mRNA compared to the HL60 cell line as expected, but lower levels compared to the healthy donors. Furthermore, MDS blasts always express higher levels of PI-PLCß1b mRNA compared to PI-PLCß1a mRNA. Our data support the contention that the deletion of the PI-PLCB1 gene is indeed responsible for a reduced expression of the enzyme. In addition,
\end{abstract}

Correspondence to: Dr Lucio Cocco, Dipartimento di Scienze Anatomiche Umane e Fisiopatologia dell'Apparato Locomotore, Sezione di Anatomia Umana, Cell Signalling Laboratory, Università di Bologna, Via Irnerio 48, I-40126 Bologna, Italy

E-mail: 1cocco@biocfarm.unibo.it

Key words: myelodysplastic syndrome, inositide-specific phospholipase $\mathrm{C} ß 1$, real-time polymerase chain reaction, chromosome the splicing isoform $1 \mathrm{~b}$, which is only nuclear, seems to be somehow partially preserved compared to the 1a isoform, which is nuclear and cytoplasmatic, hinting at a possible imbalance of the nuclear versus cytoplasmatic PI-PLC signaling which, in turn, could affect the cell cycle progression of MDS blasts.

\section{Introduction}

The myelodysplastic syndromes (MDS) are a heterogeneous group of bone marrow disorders characterized by a defect in the differentiation of the hematopoietic stem cell that causes anemia, neutropenia, bleeding problems and infections. MDS blasts are histologically analyzed and divided according to the F.A.B. (French-American-British) classification, based on the number and type of blasts present in a bone marrow aspirate and biopsy. The F.A.B. classification is useful since MDS patients can be generally divided into two major groups: patients at high and low risk of developing acute myeloid leukemia (AML). The evolution into AML involves $~ 30 \%$ of patients affected by MDS, and these patients usually have a worse clinical outcome. Even if $\sim 70 \%$ of patients affected by MDS are considered at low risk, $25-30 \%$ of them may develop AML. In addition, the prognosis of MDS patients is based on their karyotype. There are some recurrent chromosomal defects, such as trisomy of chromosome 8 or loss of chromosome $5 \mathrm{q}$, that are found in patients at major risk of developing AML, compared to patients with a normal GTG banding that are considered at minor risk (1). Phosphoinositidespecific phospholipase C (PI-PLC) B1 is a key enzyme for the nuclear signaling pathway, since it is involved in many cellular processes, such as proliferation and differentiation. It has been demonstrated that PI-PLC $\beta 1$ is down-regulated during the differentiation of Friend erythroleukemia cells (2), suggesting that this enzyme could affect the generation of MDS blasts (3). By using fluorescence in situ hybridization (FISH) analysis, the PI-PLCß1 gene has been mapped on chromosome 20p12 (4). Using the same type of probe, our group has recently studied a small number of high-risk MDS patients. Our results showed that patients bearing a monoallelic deletion of the PI-PLCß1 gene had a worse clinical outcome than patients having both alleles. To evaluate whether the disease was accompanied by reduced expression of PI-PLCß1 
mRNA, the level of PI-PLCB1 mRNA was quantified in the same patients analyzed by FISH. The alternative splicing of PI-PLCB1 produces two transcripts (1a and 1b) that differ for the presence, in the PI-PLC $\beta 1 b$ isoform, of a small exon containing a stop codon that causes the production of a shorter protein $(5,6)$. Compared to the healthy donors, MDS patients have a lower expression of both PI-PLCß1a and PI-PLCß1b. In all of the patients examined, the expression of both PIPLCB1 isoforms is higher as compared to the HL60 cell line. Furthermore, MDS blasts express higher levels of PI-PLCß1b mRNA as compared to PI-PLCß1a mRNA.

\section{Materials and methods}

Patients and cell lines. Bone marrow (BM) and peripheral blood (PB) samples were obtained from 8 patients with MDS with normal karyotype and from 10 healthy donors who had given informed consent in accordance with institutional guidelines. All samples were from the Institute of Hematology and Medical Oncology 'L. e A. Seràgnoli' of the General Hospital of Bologna. In all 8 subjects participating in this study, the diagnosis was defined according to the French American British (F.A.B.) classification, while the International Prognostic Scoring System (IPSS) was used to assess the presence of high-risk MDS (7). For in vitro experiments, bone marrow mononuclear cells (BMMCs) and peripheral blood mononuclear cells (PBMCs) were isolated by Ficoll-Paque (Amersham Biosciences, Uppsala, Sweden) density-gradient centrifugation. All analyses were performed on samples from patients at the time of diagnosis; therefore, they were not already subjected to any MDS treatment, which might affect the expression of PI-PLCß1.

HL60 cells (obtained from the American Type Culture Collection-CCL 240) were routinely maintained in RPMI1640 supplemented with $10 \%$ fetal calf serum at an optimal cell density of $3-8 \times 10^{5}$ cells $/ \mathrm{ml}$.

FISH analysis. Cytogenetic investigations were carried out on bone marrow cells stimulated for $72 \mathrm{~h}$ with phytohemagglutinin (PHA). FISH analysis was performed according to Lo Vasco et al (8) by using a specific probe for the gene of PI-PLC 31 (PAC clone HS881E24 from P de Jong RPCI-5 PAC library). As a control, we used a probe for the chromosome 20p arm subtelomeric region (Cytocell/Celbio, Italy) and one for PIPLCß4 (cDNA probe kindly provided by Dr Sue Goo Rhee, NIH, Bethesda, MD, USA). FISH analysis for PI-PLCß1 and PI-PLCB4 was performed according to standard methods; the DNA probe was biotin-labelled by nick translation and detected with Cy3-conjugated streptavidin (Sigma-Aldrich). FISH analysis for the subtelomeric region was performed according to the manufacturer's data. The images were acquired using a Nikon Eclipse 800 fluorescence microscope and the Genikon system.

RNA extraction and retro-transcription. Total cellular RNA was extracted from blasts and the HL60 cell line by using the denaturing guanidinium isothiocyanate method (Rneasy mini kit; Qiagen Ltd.) according to the manufacturer's instructions. Genomic DNA was eliminated by RNase-free DNase I digestion (Qiagen Ltd.) during the isolation procedure. cDNA
Table I. Patients' chararacteristics.

\begin{tabular}{ll} 
General & \\
No. of patients & 8 \\
Male/female ratio & $5 / 3$ \\
Median age, years (range) & $67.6(61-72)$ \\
Category & \\
High-risk MDS & \\
Cytogenetics & 8 \\
Diploid & \\
\hline
\end{tabular}

aClassified according to the IPSS.

was synthesized from $500 \mathrm{ng}$ of total RNA using $200 \mathrm{U}$ of M-MLV reverse transcriptase, $0.5 \mu \mathrm{g}$ of oligodT primers, $25 \mathrm{U}$ ribonuclease inhibitor, $10 \mathrm{mM}$ of each dNTP for $1 \mathrm{~h}$ at $42^{\circ} \mathrm{C}$.

Real-time PCR. The expression of the PI-PLCß1 gene was determined using a TaqMan based real-time PCR method. To analyze and quantify the levels of the two different isoforms of the PI-PLCß1 gene (1a and 1b), we used a TaqMan isoform specific probe (assay no. Hs01001939_m1 and Hs01008373_m1, Applied Biosystems). To establish which was the better housekeeping gene, we performed a reaction with the TaqMan human endogenous control plate according to the manufacturer's protocols and, as indicated by the plate, the glyceraldehyde-3-phosphate dehydrogenase (GAPDH) housekeeper gene (assay no. Hs99999905_m1, Applied Biosystems) was chosen as the reference. The HL60 cell line was used as another control for the real-time PCR reaction, since this cell line has a very low expression of PI-PLCß1 (9).

Amplification was performed using a total reaction volume of $25 \mu 1$ in a MicroAmp Optical 96-well reaction plate (Applied Biosystems). Real-time PCR reactions were carried out with TaqMan universal master mix (Applied Biosystems) containing the primers and probes. Reactions were run on the ABI PRISM 7300 sequence detection system (Applied Biosystems) with the following thermal conditions: $50^{\circ} \mathrm{C}$ for $2 \mathrm{~min}, 95^{\circ} \mathrm{C}$ for $10 \mathrm{~min}$ followed by 40 cycles of $95^{\circ} \mathrm{C}$ for $15 \mathrm{sec}$ and $60^{\circ} \mathrm{C}$ for $1 \mathrm{~min}$.

Quantification analysis was performed using the $\Delta \Delta \mathrm{Ct}$ method and statistically analyzed by GraphPad Prism software (v. 3.0).

\section{Results}

Patients characteristics. Bone marrow mononuclear cell (BMMC) and peripheral blood mononuclear cell (PBMC) fractions from 8 patients with normal karyotype and diagnosed with MDS were examined. Patient demographics and disease characteristics are summarized in Table I. The median age was 67.6 years (range, 61-72 years). MDS patients were classified according to the International Prognostic Score System (IPSS).

Table II refers to FISH analysis, which was performed on each MDS patient to determine the presence of PI-PLCB1, PI-PLCB4 and the 20p subtelomeric region. In particular, 3/8 
Table II. FISH analysis in high-risk MDS patients.

\begin{tabular}{|c|c|c|c|c|c|c|c|}
\hline Patients & Age & Diagnosis & Karyotype & Sample type & PLCß1 & PLCß4 & tel20p \\
\hline A & 70 & High-risk MDS & $46, \mathrm{XX}$ & Bone marrow & $+/-$ & $+/+$ & $+/+$ \\
\hline $\mathrm{B}$ & 68 & High-risk MDS & $46, X Y$ & Bone marrow & $+/+$ & $+/+$ & $+/+$ \\
\hline $\mathrm{C}$ & 65 & High-risk MDS & $46, \mathrm{XX}$ & Bone marrow & $+/+$ & $+/+$ & $+/+$ \\
\hline $\mathrm{D}$ & 72 & High-risk MDS & $46, X Y$ & Bone marrow & $+/-$ & $+/+$ & $+/+$ \\
\hline $\mathrm{E}$ & 71 & High-risk MDS & $46, \mathrm{XX}$ & Bone marrow & $+/+$ & $+/+$ & $+/+$ \\
\hline $\mathrm{F}$ & 64 & High-risk MDS & $46, X Y$ & Peripheral blood & $+/-$ & $+/+$ & $+/+$ \\
\hline G & 70 & High-risk MDS & $46, X Y$ & Peripheral blood & $+/+$ & $+/+$ & $+/+$ \\
\hline $\mathrm{H}$ & 61 & High-risk MDS & $46, X Y$ & Peripheral blood & $+/+$ & $+/+$ & $+/+$ \\
\hline
\end{tabular}

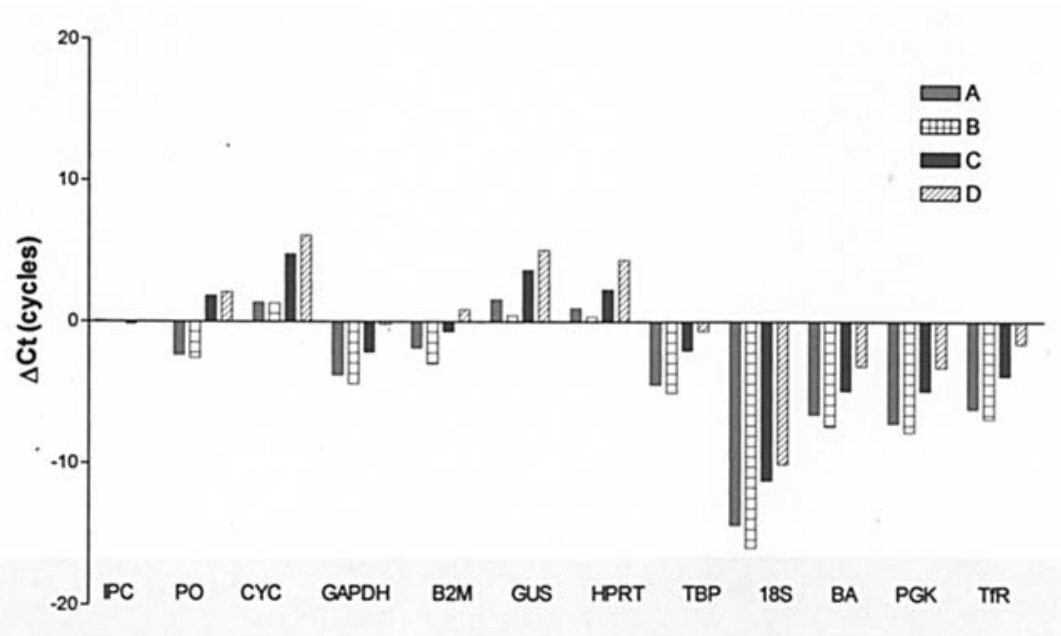

A

Figure 1. TaqMan endogenous plate control analysis in healthy donors and high-risk MDS patients. The analysis was performed in three healthy donors (samples A, B and C) and a high-risk MDS patient (sample D). (A) Bars represent the difference for each gene compared to the internal positive control (IPC) in all of the samples examined (A-D). (B) The table represents the mean values of the difference for each gene compared to the IPC. The 11 genes analyzed were from the most stable to the least stable, as compared to the internal positive control (IPC): B2M (ß-2-microglobulin), GAPDH (glyceraldehyde-3phosphate dehydrogenase), TBP (TATA-binding protein), PO (acidic ribosomal protein), TfR (transferrin receptor), HPRT (hypoxanthine phosphoribosyl transferase), BA (ß-actin), PGK (phosphoglycerokinase), GUS (ß-glucuronidase), CYC (cyclophilin), 18S rRNA.

high-risk MDS patients (38.5\%) showed monoallelic deletion of the PI-PLCß1 gene, while both PI-PLCB4 and the 20p subtelomeric region were normal in all patients.

Reference genes for real-time PCR. The internal reference was chosen according to the results obtained from the TaqMan human endogenous plate control (Fig. 1), where 11 commonly used housekeeping genes are investigated. The analysis was performed on isolated blasts from three healthy donors (samples A, B and C) and one patient (sample D). As Fig. 1 shows, the range of expression stability of these genes was, from the most stable to the least stable: B2M (B-2-microglobulin), GAPDH (glyceraldehyde-3-phosphate dehydrogenase), TBP (TATA-binding protein), PO (acidic ribosomal protein), TfR (transferrin receptor), HPRT (hypoxanthine phosphoribosyl transferase), BA (B-actin), PGK (phosphoglycerokinase), GUS (ß-glucuronidase), CYC (cyclophilin), 18S rRNA.
The two genes showing the least variance and which could, therefore, be used as internal reference were B2M and GAPDH. Even though the B2M gene has been used as an internal reference gene for real-time PCR reactions in peripheral blood cells (10), we decided to use GAPDH because it is unrelated to the hematopoietic system.

PLCß1a and PLCß1b mRNA expression in healthy donors and the HL60 cell line. mRNAs from 10 healthy donors' BMMC and PBMC fractions were extracted and retrotranscribed. Then, we performed a real-time PCR reaction in order to determine the absolute amount of PI-PLCB1a and PI-PLCß1b transcript in these samples (data not shown). The cDNAs were subsequently mixed considering the results obtained from this analysis and used as the calibrator for the MDS patients analysis.

The HL60 cell line was used as another internal control for the method, since this cell line has a very low expression 

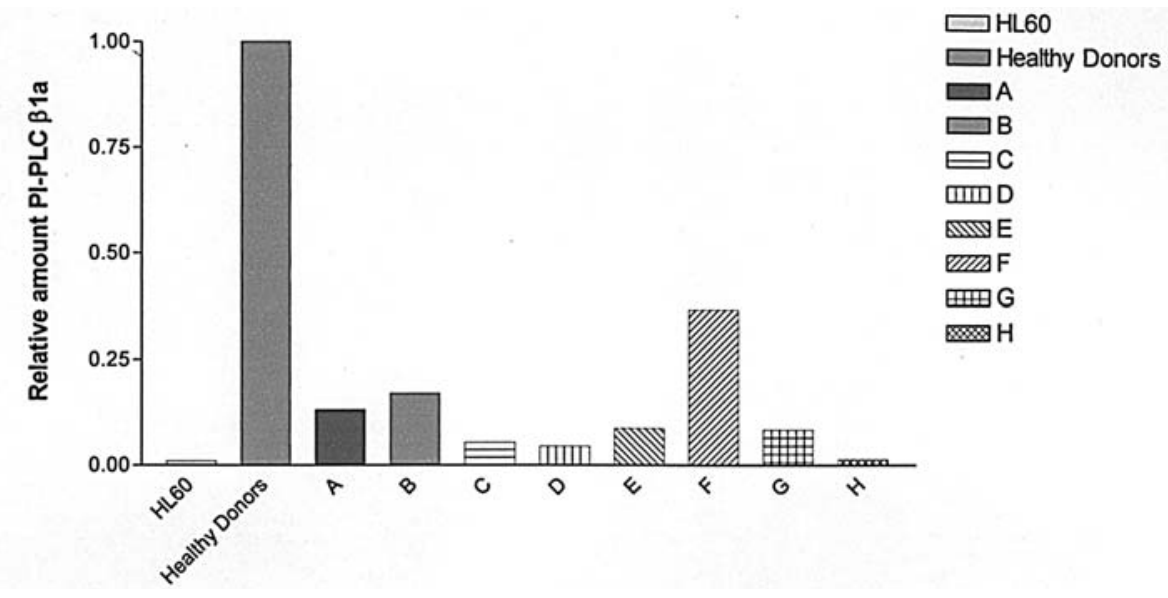

Figure 2. Relative quantification of PI-PLCß1a mRNA in high-risk MDS patients. All MDS patients (A-H) showed significant reduced gene expression compared to healthy donors and a higher expression compared to the HL60 cell line. GAPDH was used as a housekeeping gene for all the samples. The fold difference evaluated by $\Delta \Delta \mathrm{Ct}$ method was calculated from three different experiments and statistically analyzed by a Dunnet test after ANOVA. All the relative amounts were statistically different ( $\mathrm{p}<0.01$ vs. healthy donors).

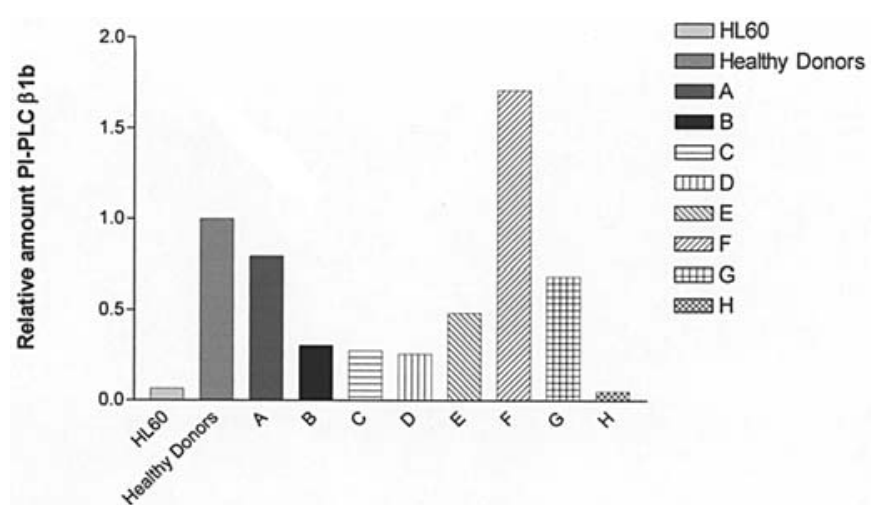

Figure 3. Relative quantification of PI-PLCß1b mRNA in high-risk MDS patients. All of the MDS patients (A-H) showed significant altered gene expression compared to healthy donors and a higher expression compared to the HL60 cell line. The high-risk MDS patients had a lower amount of PIPLCB1b mRNA, except for patient F, who showed a higher amount of mRNA. GAPDH was used as a housekeeping gene for all of the samples. The fold difference evaluated by $\Delta \Delta \mathrm{Ct}$ method was calculated from three different experiments and statistically analyzed by a Dunnet test after ANOVA. All the relative amounts were statistically different ( $\mathrm{p}<0.01$ vs. healthy donors).

of the PI-PLCß1 transcripts. In fact, by using the PLC $\beta 1$ amount of the healthy donors as the calibrator (100\%), the HL60 cell line had the lowest expression of both the PI-PLCß1 isoforms, showing a decrease of $99 \%$ for the PI-PLCB1a isoform and a PI-PLCß1b mRNA level of $6 \%$ compared to the healthy donors (Figs. 2 and 3).

PLCß1a and PLCß1b mRNA expression in MDS patients. The results obtained with the PI-PLCß1a probe show that high-risk MDS patients have a lower level of the transcript compared to the healthy donors (Fig. 2). Considering a value of $100 \%$ for healthy donors, the relative amount of PLCB1a mRNA in all of the patients is comprised between 2 and 35\%, as shown in Fig.2 .

Similarly, with the PI-PLCß1b probe, most of the highrisk MDS patients $(90 \%)$ demonstrated a lower expression of the PI-PLCB1b mRNA (Fig. 3). In this case, the samples show more variability in PI-PLC $11 \mathrm{~b}$ relative amount, since the mRNA levels vary from 5 to $94 \%$ (healthy donors=100\%).

By comparing the two assays results, we saw that all of the patients show an increase in the relative amount of the PIPLCß1b transcript as compared to the PI-PLCß1a isoform (Fig. 4).

\section{Discussion}

Recently, we have seen that patients affected by myelodysplastic syndrome and bearing a monoallelic deletion in the PI-PLCB1 gene have a worse clinical outcome than patients with both alleles (8). Even though this feature applies to high-risk and not to all MDS patients, it is interesting because of the fact that the PI-PLCß4 gene, which is located closely to PI-PLCß1, is unaffected, showing that we are facing a cryptic deletion in patients with a normal GTG banding karyotype $(8,11)$. Thus, we decided to analyze further the molecular biology of this deletion.

To determine whether the monoallelic deletion of the PIPLCB1 gene results in reduced gene expression, we investigated the PI-PLCB1 mRNA levels in these patients. The relative expression of the PI-PLCß1 gene was studied by means of real-time PCR. The study involved both healthy donors and patients affected by myelodysplastic syndrome and formerly analyzed by FISH. The blast cells were from MDS patients (with a normal karyotype) at high risk of developing AML, all at the time of MDS diagnosis (Table I). We used specific probes for the two transcripts of the PI-PLCß1 gene (1a and 1b). The analysis was performed through a semi-quantitative method, with the average transcript amount of healthy donors as a calibrator. The GAPDH gene was the internal reference for each sample, useful for determining any sample-to-sample variation of the quantity and quality of RNA and differences in efficiency of reverse transcription and PCR reactions.

Our results show that the expression of PI-PLCß1 mRNA is altered in patients affected by MDS at high risk of evolution into AML. In fact, all of the patients analyzed showed a 


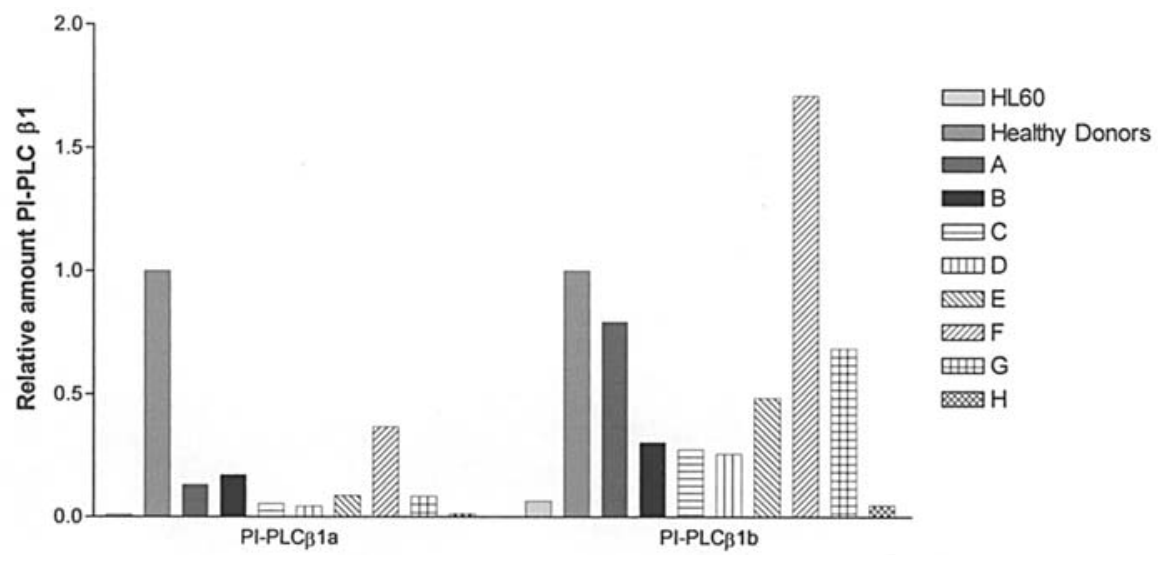

Figure 4. Comparison of the relative amount of PI-PLCß1a and PI-PLCß1b mRNA in high-risk MDS patients. PI-PLCß1a and PI-PLCß1b mRNA relative amount in high-risk MDS patients. PI-PLCß1b expression is always higher, compared to that of the PI-PLCß1a isoform.

decrease in the amount of PI-PLCß1a. PI-PLCß1b expression was also affected since, in almost all of the high-risk patients, we found a lower level of this isoform. Collectively, our data suggest that a reduced expression of PI-PLC 1 mRNA is frequently present in patients affected by high-risk MDS.

Interestingly, MDS cells always express higher levels of PI-PLCß1b mRNA compared to PI-PLCß1a mRNA. This difference could reflect a specific effect of the different localization of these two mRNA transcripts. In fact, splicing isoform 1a demonstrates both nuclear and cytoplasmatic localization, while splicing isoform $1 \mathrm{~b}$ is localized only to the nucleus. The same difference was found between the amount of the two isoforms in the healthy donors.

The altered expression of nuclear PI-PLCß1 mRNA could be related to an altered expression of the protein and, as a consequence, it could alter the nuclear lipid signaling pathway, which is involved in different processes, such as cell proliferation and differentiation. In particular, as reported by Faenza et al (12), nuclear PI-PLCß1 can induce cell cycle progression in Friend erythroleukemia cells. As a consequence, cyclin D3, along with its kinase (cdk4), is activated in a specific manner. Moreover, these authors showed that retinoblastoma protein is phosphorylated and E2F-1 transcription factor is activated, indicating a direct effect of nuclear PLCß1 signaling in G1 progression by means of a specific target, i.e. cyclin D3/cdk4. In conclusion, these findings could have an important meaning for high-risk MDS patients, since the altered expression of nuclear PI-PLCß1 could be involved in a disregulation of the cell cycle and also have important effects on cell apoptosis pathways.

\section{Acknowledgements}

This work was supported by AIRC, Italian Ministry of University and Research FIRB and Cofin grants and CARISBO Foundation.

\section{References}

1. Mori N, Morosetti R, Hoflehner E, Lubbert M, Mizoguchi H and Koeffler HP: Allelic loss in the progression of myelodysplastic syndrome. Cancer Res 60: 3039-3042, 2000.

2. Martelli AM, Billi AM, Gilmour RS, Neri LM, Manzoli L, Ognibene A and Cocco L: Phosphoinositide signaling in nuclei of Friend cells: phospholipase C beta down-regulation is related to cell differentiation. Cancer Res 54: 2536-2540, 1994.

3. Cocco L, Manzoli L, Palka G and Martelli AM: Nuclear phospholipase $C$ beta1, regulation of the cell cycle and progression of acute myeloid leukemia. Adv Enzyme Regul 45: 126-135, 2005.

4. Peruzzi D, Calabrese G, Faenza I, Manzoli L, Matteucci A, Gianfrancesco F, Billi AM, Stuppia L, Palka G and Cocco L: Identification and chromosomal localisation by fluorescence in situ hybridisation of human gene of phosphoinositidespecific phospholipase C beta(1). Biochim Biophys Acta 1484: $175-182,2000$.

5. Bahk YY, Song H, Baek SH, Park BY, Kim H, Ryu SH and Suh PG: Localization of two forms of phospholipase C-beta1, a and $\mathrm{b}$, in C6Bu-1 cells. Biochim Biophys Acta 1389: 76-80, 1998.

6. Cocco L, Manzoli L, Barnabei O, Gilmour RS and Martelli AM: Re-examination of the significance of nuclear localization of PLCbeta1 in the likelihood of its involvement in neoplastic cell growth. Adv Enzyme Regul 43: 1-13, 2003.

7. Greenberg P, Cox C, LeBeau MM, Fenaux P, Morel P, Sanz G, Sanz M, Vallespi T, Hamblin T, Oscier D, Ohyashiki K, Toyama K, Aul C, Mufti G, and Bennett J: International scoring system for evaluating prognosis in myelodysplastic syndromes. Blood 89: 2079-2088, 1997.

8. Lo Vasco VR, Calabrese G, Manzoli L, Palka G, Spadano A, Morizio E, Guanciali-Franchi P, Fantasia D and Cocco L: Inositide-specific phospholipase c betal gene deletion in the progression of myelodysplastic syndrome to acute myeloid leukemia. Leukemia 18: 1122-1126, 2004.

9. Lukinovic-Skudar V, Donlagic L, Banfic H and Visnjic D: Nuclear phospholipase C-beta1b activation during G2/M and late G1 phase in nocodazole-synchronized HL-60 cells. Biochim Biophys Acta 1733: 148-156, 2005.

10. Oselin K, Mrozikiewicz PM, Pahkla R and Roots I: Quantitative determination of the human MRP1 and MRP2 mRNA expression in FACS-sorted peripheral blood CD4+, CD8+, CD19+, and CD56+ cells. Eur J Haematol 71: 119-123, 2003.

11. Lo Vasco VR, Follo MY and Cocco L: Reply to Herens et al. Leukemia 20: 522-523, 2006.

12. Faenza I, Matteucci A, Manzoli L, Billi AM, Aluigi M, Peruzzi D, Vitale M, Castorina S, Suh PG and Cocco L: A role for nuclear phospholipase Cbeta 1 in cell cycle control. J Biol Chem 275: 30520-30524, 2000. 